\title{
INFORMAÇÃO E CONHECIMENTO: INSUMOS PARA O TRABALHO ORGANIZATIVO EM CIÊNCIA DA INFORMAÇÃO
}

\author{
INFORMATION AND KNOWLEDGE: INPUTS FOR \\ ORGANIZATIONAL WORK IN INFORMATION SCIENCE
}

Giovana Deliberali Maimonea

\begin{abstract}
RESUMO
Introdução: Conceitua informação e conhecimento no campo da Ciência da Informação para elucidar a importância que adquirem ao integrarem Sistemas de Organização do Conhecimento (SOC). Objetivo: Evidenciar a importância dos conceitos de informação e conhecimento para os SOC. Metodologia: Levantamento e análise de referencial teórico sobre a temática da organização da informação e do conhecimento e os sistemas de organização do conhecimento (SOC). Resultados: Informação e conhecimento são elementos fundamentais para a elaboração de sistemas de organização do conhecimento (SOC) pois a informação é veículo comunicativo no âmbito social e o conhecimento além de ser portador de desenvolvimento científico e tecnológico, quando registrado é passível de transformação em informação documentária. Os SOC estruturam-se em linguagens controladas, ferramentas propositalmente elaboradas para acesso à informação. Conclusões: Todo o sistema organizativo em Ciência da Informação passa necessariamente por algum tipo de linguagem que pode proporcionar recuperação da informação para se transformar em conhecimento.
\end{abstract}

Descritores: Informação. Conhecimento. Sistemas de Organização do Conhecimento.

\section{INTRODUÇÃO}

Os conceitos de informação e conhecimento são abordados de modo específico no campo da Ciência da Informação a fim de justificar atividades que integram a "Organização do Conhecimento" (OC) e os Sistemas de Organização do Conhecimento (SOC) que dela derivam. Tais sistemas revelam fortes relações com a Linguística e a Terminologia já que estão fundamentados na estrutura da Linguagem, valendo-se, estas últimas, de algum sistema de códigos para torná-lo funcional.

\footnotetext{
a Doutora em Ciência da Informação pela Escola de Comunicações e Artes da Universidade de São Paulo (USP). Professora da Universidade de São Paulo (USP) no curso de Informação e Cultura (CBD). E-mail: gdmaimone@usp.br.
} 
Segundo Lyons (1977, p. 35) "Os principais sistemas de sinais empregues pelo ser humano para a transmissão de informação, se bem que não sejam os únicos, são as línguas". A codificação pode ser numérica como são os sistemas de classificação que intentam auxiliar na localização física do documento; verbal, que utilizam termos "preferidos" para controlar a indexação e recuperação do acervo (tesauros); imagética que propõem a elaboração de mapas conceituais para a "demonstrar" o conhecimento de modo mais imediato, entre outros.

O uso das linguagens documentárias no cenário da Ciência da Informação intenta resolver problemas como polissemia, ambiguidade, redundância com o objetivo de possibilitar a recuperação da informação de forma padronizada. À título de exemplificação, o tesauro, tipo específico de linguagem controlada, é estruturado a partir de definições de termos preferidos e não preferidos, ordens hierárquicas entre conceitos e associações possíveis; um conceito para apenas um termo e um termo para apenas um conceito. Assim os sistemas de organização do conhecimento têm como "objetivo essencial agrupar informações semelhantes e diferenciar as que são diferentes" (SVENONIUS, 2001, p. 11).

Além desta pequena introdução o artigo tratará especificamente da Organização do Conhecimento e suas ocorrências conceituais, estabelecendo relações diretas com os Sistemas de Organização do Conhecimento, para em seguida revelar questões específicas sobre as linguagens utilizadas para este fim.

\section{ORGANIZAÇÃO DO CONHECIMENTO E INFORMAÇÃO}

A organização do conhecimento está voltada ao estudo de processos que possibilitem o acesso aos registros do conhecimento (documentos), a fim de que se tenha uma recuperação informacional adequada dos itens buscados.

Em um primeiro momento distingue-se informação de conhecimento por sua própria natureza, ou seja, a informação é de natureza social, circula entre pessoas e grupos, funciona como fluxo e permite a comunicação. Já o conhecimento é construído individualmente e advém da elaboração mental subjetiva do indivíduo. Nesse sentido, a informação pode ser compreendida como matéria-prima do conhecimento, pois a partir dela é possível sua geração, 
alimentando o ciclo social da informação apresentado por Le Coadic (2004) que permite o desenvolvimento científico, social e tecnológico. $O$ trabalho de "organização" (da informação e do conhecimento) possibilita tal fluxo pelo tratamento documentário realizado por meio dos documentos de partida.

Ainda com o intuito de conceituar o termo informação no domínio da Ciência da Informação, Le Coadic (2004, p.4) apresenta: "A informação é um conhecimento inscrito (registrado) em forma escrita (impressa ou digital), oral ou audiovisual, em um suporte". Representar informação advinda de um registro do conhecimento, ou seja, dos documentos, é tarefa fundamental para o adequado funcionamento dos sistemas de recuperação de informação. Por este motivo, é preciso garantir fidedignidade ao material tratado, oferecendo-Ihe autenticidade, já que de tal processo pode originar novos conhecimentos.

[...] o uso da palavra informação indica uma perspectiva específica, a partir da qual o conceito de comunicação do conhecimento tem sido definido. Esta perspectiva inclui características como novidade e relevância, ou seja, refere-se ao processo de transformação do conhecimento $e$, particularmente, à seleção e interpretação dentro de um contexto específico (CAPURRO, HJØRLAND, 2007, p. 150).

Todo documento relevante para o usuário é fonte potencial de informação geradora de conhecimento. Apesar da interpretação ficar por conta do usuário, o bibliotecário pode auxiliar na busca dos materiais constantes do acervo indicando pistas de acesso ${ }^{1}$ que indiquem os documentos de modo objetivo, auxiliando na efetividade comunicativa. Segundo Saracevic (1975, p. 321) "In the most fundamental sense, relevance has to do with effectiveness of communication".

Para Barreto (2005), a informação pode ser considerada como uma ferramenta de modificação da consciência dos indivíduos, pois é o elemento essencial da transferência de conteúdo. Desse modo, desempenha uma função mediadora na produção do conhecimento, porém, a informação somente exercerá sua função de gerar/complementar conhecimento quando assimilada e compreendida como tal, ou seja,

Quando usamos o termo informação em Ciência da Informação, deveríamos ter sempre em mente que informação é o que é

\footnotetext{
${ }^{1}$ Descritivas ou temáticas.
} 
informativo para uma determinada pessoa. O que é informativo depende das necessidades interpretativas e habilidades do indivíduo (embora estas sejam frequentemente compartilhadas com membros de uma mesma comunidade de discurso) (CAPURRO; HJØRLAND, 2007, p. 154 - 155).

A “apropriação" da informação deve diferir, portanto, de pessoa para pessoa, considerando que "uma mesma informação pode ter diversos significados para diferentes pessoas e para a mesma pessoa em diferentes tempos (BARRETO, 2005, p. 7)". Isso devido à interpretação que cada um de nós realiza sobre determinada informação; na realidade é um ato de selecionar entre as possibilidades semânticas e pragmáticas de uma mensagem (CAPURRO; HJØRLAND, 2007), que depende do contexto e dos graus de limitação dos usuários.

Independentemente do uso (ou não) que se faça da informação, intentase evidenciar o papel fundamental que a Ciência da Informação desempenha no que se refere à sua função mediadora a partir da representação documentária, tornando, nesta perspectiva, mais democrático o acesso à informação.

Ocupando-se das questões informacionais com enfoque prioritário nos públicos utilizadores, concorda-se com Paul Otlet, que a informação deve tratar de fenômenos substancialmente sociais, uma vez que nasce na sociedade e a ela deve retornar (KOBASHI; TÁLAMO, 2003). É válido recordar a visão de Farradane, de que

[...] a informação deve ser entendida como algo físico: qualquer forma física de representação ou substituto do conhecimento ou de um pensamento particular, e utilizada para comunicação (BAWDEN, 2008, p. 419).

A informação e o conhecimento sempre estiveram atrelados ao poder e, por muito tempo, ficaram sob a "guarda" e a preservação de poucos, o que implicou "menores possibilidades de circulação social da informação" (LARA, 2007, p. 153).

Para Zins (2004), existem duas abordagens básicas para definir o conceito de conhecimento das quais compartilhamos: o conhecimento como um pensamento do indivíduo ou do sujeito, e o conhecimento como objeto ou coisa. O primeiro aborda condições do conhecimento na mente do indivíduo.

Conhecimento é um pensamento que pode ser caracterizado como uma crença verdadeira justificada. [...] O segundo [...] é 
uma coleção de conceitos, argumentos, argumentações, e regras de inferência. Ele é verdadeiro e existe independentemente, não dependendo do conhecimento subjetivo ou individual (ZINS, 2004, p. 50).

Esse mesmo autor prossegue em suas considerações ao propor a organização do conhecimento sob uma perspectiva epistemológica concluindo que todo conhecimento é produto de uma síntese, diferenciando o conhecimento empírico do teórico.

O conhecimento empírico é o produto de uma síntese de impressões empíricas ou conceitos pré-experimentais. Conhecimento teórico é o produto de uma síntese do material intelectual de alto nível de conceitos (ZINS, 2004, p. 52).

Tais exposições remetem a reflexões sobre algumas teorias propostas pela Ciência Cognitiva, já que esta explora estudos sobre o funcionamento da mente humana. Revela-se então a existência de graus de conhecimento que segundo Platão e Aristóteles, se dividiam em dois: conhecimento sensível e conhecimento inteligível, de modo que o primeiro compreenderia crenças e opiniões e o segundo raciocínios e intuições intelectuais (CHAUÍ, 2009).

Para a Ciência da Informação, o interesse recai sobre o nível do conhecimento inteligível, pois trabalha-se com o conhecimento registrado, e geralmente embasado em estudos científicos (campo intelectual). Contrariamente ao sensível (ilusório), o conhecimento inteligível pode ser considerado conhecimento verdadeiro.

O pensamento é inerente à espécie humana, e permite, graças à assimilação de informações, a geração de conhecimentos, intermediados por símbolos conhecidos. Por esse motivo, a representação informacional de documentos é imprescindível para a transmissão da cultura através da linguagem. Faz sentido então relembrar a equação fundamental da Ciência da Informação, destacando que

A absorção de informações em uma estrutura de conhecimento não pode causar uma simples adição, mas algum ajuste na estrutura, como uma mudança nas relações ligando dois ou mais conceitos já admitidos (BROOKES, 1980, p. 131).

O conhecimento é uma estrutura de conceitos ligados por suas relações e a informação é apenas uma pequena parte de tal estrutura (ZINS, 2004). Todo sistema de informação deve estar pautado nos objetivos institucionais e também 
na possibilidade de acesso e recuperação dos materiais buscados. Sob essa ótica, Belkin (1980) propõe pensar a recuperação da informação focada no usuário, ou melhor, no estado anômalo do conhecimento, trabalhando a questão da pergunta do usuário ao sistema em relação à informação desejada.

\begin{abstract}
A discussão neste documento será limitada a uma visão muito particular de recuperação de informação (IR). Minha premissa básica é que a Ciência da Informação, e, portanto, IR, é uma disciplina orientada para o problema, preocupada com 0 problema da transferência eficaz e eficiente da informação desejada entre o gerador humano e o usuário humano (BELKIN, 1980 , p. 133).
\end{abstract}

Segundo Afzal e Thompson (2011), apesar da insistência dos estudos centrados apenas nos usuários (modelos mentais individuais), deve-se reconhecer que a ênfase sociocognitiva oferece mais suporte às pesquisas para a recuperação da informação, por enfatizar a importância do contexto e do conhecimento em uma percepção social mais ampla.

Além, contudo, de estar focalizada nas necessidades dos usuários e na relevância do sistema, é também proeminente pensar que os sistemas de informação devem atender públicos com necessidades distintas, fato que amplia a noção cognitiva ou sociocognitiva para uma concepção amparada em investigações culturais. Assim, a visão cognitiva é fortemente abalada pelas "abordagens mais hermenêuticas e comportamentais para a transferência de informação" (INGWERSEN, 1992, p. 299).

\title{
3 SISTEMAS DE ORGANIZAÇÃO DO CONHECIMENTO, INFORMAÇÃO E LINGUAGEM
}

Embora ainda não haja consenso entre os pesquisadores da área da Ciência da Informação sobre os termos organização da informação e organização do conhecimento, Bräscher e Café (2010), afirmam haver uma diferenciação entre o trabalho efetuado sobre informações e aquele realizado sobre o conhecimento. "O objetivo do processo de organização da informação é possibilitar o acesso ao conhecimento contido na informação" (BRÄSCHER; CAFÉ, 2010, p. 91).

Assim, a organização da informação seria o processo que abrange 
atividades sobre os registros do conhecimento, ou seja, descrevem-se características físicas e de conteúdo dos documentos, sendo sua representação o produto dessas atividades, ou seja, o conjunto de atributos que representam determinado objeto informacional.

Já o processo de organização do conhecimento se aplicaria às unidades do pensamento, ou seja, aos conceitos. Em outras palavras, seria o modo de modelar o conhecimento que aponta para a construção de representações (BRÄSCHER; CAFÉ, 2010).

Os sistemas de organização do conhecimento (SOC) são ferramentas que permitem arranjar os documentos ou, representar o conhecimento, a partir de certa ordem, previamente estipulada.

Tomando como base o conceito de que todo sistema de organização do conhecimento apresenta-se forçosamente como estrutura e representação (TÁLAMO, 1997), Roqueta (2011) expõe treze tipos diferentes de sistemas de organização do conhecimento que têm como elementos comuns a estrutura (de conceitos) e uma forma de representação própria (sistematização do conhecimento acumulado). Segundo este mesmo autor, os traços essenciais de sistemas de organização do conhecimento são:

Sua referência ao conhecimento especializado;

Sua estrutura lógica, que se constrói de acordo com um método e uma teoria de organização; e,

O controle de vocabulário, que contribui para a seleção, depuração, formalização e normalização da terminologia (ROQUETA, 2011, p. 127).

A criação de mecanismos para "organizar" e "representar" o conhecimento permite a identificação de termos/conceitos e a análise de suas características, possibilitando o estabelecimento de relações. Trabalhar com documentos envolve análise, interpretação e representação do material, construindo um novo tipo de "apresentação" chamada informação documentária. Tais ações devem ser realizadas de acordo com os objetivos da instituição, tomando como base a linguagem dos usuários e dos materiais.

A Representação da Informação, produto da análise dos documentos de cada área, e das reflexões sobre a realidade que se pretende representar é realizada por meio de sistemas de organização do conhecimento que integram 
uma estrutura conceitual e suas relações lógico-semânticas com o intuito de representar modelos de mundo.

A Organização do Conhecimento propõe "instrumentos" de representação que possibilitam o tratamento do conteúdo, executando atividades que se situam na intermediação da solicitação do usuário em relação à coleção da instituição, com a finalidade de proporcionar adequada recuperação da informação. Sob essa ótica, concorda-se com Hjorland (2008, p. 99), quando defende que a "Organização do Conhecimento [...] tem por objetivo apoiar a aprendizagem e as atividades de pesquisa", disponibilizando acesso aos documentos e fomentando o interesse científico, artístico e cultural.

Segundo Broughton et al. (2005), as funções básicas dos sistemas de organização do conhecimento são: facilitar a recuperação da informação; fornecer informações sobre documentos e efetuar o arranjo das prateleiras (ordenação). Para auxiliar tais trabalhos, Hodge (2000), apud Friedman e Thellefsen (2011, p. 646) divide os tipos de sistemas de organização do conhecimento em três categorias principais, de forma a fornecer uma visão útil das mesmas, são elas:

1. Listas de termos: arquivos de autoridade, glossários, dicionários e outros.

2. Classificações e categorias: cabeçalhos de assunto, esquemas de classificação, taxonomias e esquemas de categorização.

3. Listas de relacionamentos: tesauros, redes semânticas e ontologias (HODGE, 2000 apud FRIEDMAN; THELLEFSEN, 2011, p. 646).

Fortalecendo as afirmações acima, pode-se dizer que os sistemas de organização do conhecimento e o efetivo sucesso da recuperação informacional dependem de diversos fatores, dentre eles a elaboração de representações documentárias diretamente relacionadas à articulação linguística.

Levando em consideração a recuperação informacional teremos dois grandes eixos: o do usuário e seu problema de pesquisa e dos documentos (conhecimento registrado). Interessa especialmente reconhecer que as representações textuais são informações retiradas do próprio documento que permitem acessar sua localização através de, por exemplo: nome de autor, título do texto, data da obra, assunto. Técnicas de recuperação da informação em 
sistemas dizem respeito aos sistemas de busca como a lógica booleana por exemplo. Às funções intermediárias cabe o reconhecimento da solicitação do usuário, sua "tradução" para a linguagem do sistema de organização do conhecimento (tesauros, vocabulários controlados, listas de cabeçalhos de assuntos) e a busca pelo material desejado tendo como base a linguagem de indexação utilizada. Em outros termos, a função da linguagem controlada está vinculada à efetiva comunicação e recuperação da informação evidenciada pela possibilidade de representar de modo "unívoco" o acervo de determinada instituição.

"No sentido mais corrente, língua é um instrumento de comunicação, um sistema de signos vocais específicos aos membros de uma mesma comunidade" (DUBOIS, 1973, p. 378) [o grifo é do autor]. Tomando por base tal posicionamento, infere-se que são fundamentais o compartilhamento e as relações desse "sistema de signos", que reafirma um dos princípios essenciais da linguística moderna, enunciado por Ferdinand de Saussure,

Em uma língua, um signo só se define como tal no seio de um conjunto de outros signos. Ele tira seu valor e seu rendimento das oposições que contrai com eles. Um signo se define, portanto, por suas relações com aqueles que o envolvem [...] (DUBOIS, 1973, p. 379).

A língua é tida como um "sistema supra-individual, na medida em que ela é definida não por um indivíduo, mas pelo grupo social a que pertence" (LOPES, 1993, p. 77). A função mediadora da língua parece óbvia, permitindo a assimilação, perpetuação ou transformação da cultura, já que é por intermédio dela que se organizam os sistemas simbólicos pelos quais cada sociedade se identifica (BENVENISTE, 1991). Portanto, a linguagem verbal é matéria do pensamento e veículo da comunicação social (PETTER, 2003).

Por sua capacidade de causar tamanhas transformações, a linguagem se faz objeto de estudo de diversos pesquisadores que se dedicaram a arte de investigá-la, tomando-a como produtora e transformadora das consciências humanas (MAIMONE, 2012, p. 35).

Sob o olhar da Semiótica, o signo deve "ocupar o lugar de algo", já que exerce a função de representar determinada "coisa". Exemplificando, a fotografia de uma igreja, assim como a palavra igreja são signos (diferentes) do mesmo "objeto material". A linguagem é apresentada como faculdade simbólica que 
revela o poder criativo e constitutivo da cultura informacional, pois,

[...] além do seu funcionamento codificador, ela se propõe como representação, seja como insumo do processo social de geração de sentido, seja como resultado textual desse processo (TÁLAMO; LARA, 2006, p. 205).

Tendo como base a ação de representação informacional através da linguagem verbal, é fundamental citar Jean-Claude Gardin, primeiro teórico a identificar uma trajetória de interdisciplinaridade entre Documentação e Linguística. Gardin, em 1973, já identificava algumas contribuições que a Linguística poderia oferecer para a análise de documentos, principalmente em relação às "ferramentas intelectuais" usadas pelos documentalistas, como eram os sistemas de classificações, as listas de termos indexadores, tesauros etc. Recordando alguns dos princípios mais respeitáveis da teoria linguística, Gardin expõe que:

a) O mais visível é a prioridade dada à análise sintática, considerada um passo necessário para iniciar a descrição ou geração do texto em linguagem natural.

b) [...] a análise semântica está confinada a uma posição controlada [...], que ainda parece ser a base para a ideia de que as propriedades da "estrutura superficial" desempenham um papel principal na determinação do significado.

c) Um terceiro princípio, também relacionado com o primeiro, é que a unidade-padrão de análise deve ser o período em que é mais ou menos de forma intuitiva definido na gramática tradicional: qualquer unidade mais ampla é sentida para ultrapassar o âmbito da análise sintática.

d) Da mesma forma, os ingredientes básicos da nova teoria permanecem nas categorias gramaticais (substantivos, verbos, adjetivos etc.) e suas funções (sujeito, objeto etc.) dos tempos antigos, que são mais refinados, mas não melhor fundados a priori para a expressão de sentido, etc. (GARDIN, 1973, p. 153 - 154) [o grifo é do autor].

Observa-se que tais princípios estão intimamente ligados com a análise de documentos, pois são utilizados também para representar materiais através das funcionalidades da língua e da possibilidade de categorização. Sob este aspecto é evidente a contribuição da área da Linguística para resultados mais satisfatórios da representação documentária. 


\section{CONSIDERAÇÕES FINAIS}

Informação e conhecimento são elementos fundamentais para a elaboração de sistemas de organização do conhecimento (SOC) pois a informação é veículo comunicativo no âmbito social e o conhecimento além de ser portador de desenvolvimento científico e tecnológico, quando registrado é passível de transformação em informação, documentária.

A sociedade contemporânea passa por um momento de interação informacional quase que totalmente digital, em que as atividades e os suportes para elaboração de tarefas estão vinculados diretamente à tecnologia comunicacional, muito atrelados ao desenvolvimento dos dispositivos móveis. Apesar de grande avanço tecnológico assistido durante todo o século passado e mais intensamente neste século uma questão fundamental não foi sanada, aliás vem aumentando com as possibilidades de acesso remoto e a uma grande quantidade de informação: o modo como a informação é recebida e se é decodificada de forma adequada para ser entendida por seu público.

Evidentemente refere-se a uma preocupação recorrente aos estudos da linguagem que procura intermediar e "traduzir" de forma clara e fidedigna a informação contida no conhecimento registrado. É para este fim que os SOC são pensados de modo integrado ao acervo da instituição e às necessidades dos usuários, estruturando-se em linguagens controladas, ferramentas propositalmente elaboradas para acesso à informação. Todo o sistema organizativo em Ciência da Informação passa necessariamente por algum tipo de linguagem que deve proporcionar recuperação da informação para se transformar em conhecimento.

\section{REFERÊNCIAS}

AFZAL, W.; THOMPSON, K. M. Contributions of cognitive science to information science: an analytical synopsis. Emporia State Research Studies, v. 47, n. 1 , p. $18-23,2011$.

BARRETO, A. de A. A estrutura do texto e a transferência da informação.

Datagramazero - Revista de Ciência da Informação, Rio de Janeiro, v. 6, n. 3, jun. 2005. 
BAWDEN, D. Smoother pebbles and the shoulders of giants: the developing foundations of information science. Journal of Information Science, v. 34, n. 4, p. $415-426,2008$.

BELKIN, N. J. Anomalous States of Knowledge as a Basis for Information Retrieval. The Canadian Journal of Information Science, v. 5, p. 133 - 143, 1980.

BENVENISTE, É. Vista d`olhos sobre o desenvolvimento da linguística. In: 1991. Problemas de linguística geral, I. São Paulo: Ed. Nacional; EDUSP,

BRÄSCHER, M.; CAFÉ, L. Organização da Informação ou Organização do Conhecimento? In.: LARA, M. L. G. de; SMIT, J. W. (org.). Temas de pesquisa em Ciência da Informação no Brasil. São Paulo: Escola de Comunicações e Artes / USP, 2010. p. 85 - 104.

BROOKES, B. C. The foundations of information Science: part 1 - philosophical aspects. Journal of Information Science, v. 2, p. $125-133,1980$.

BROUGHTON, V.; HANSSON, J.; HJØRLAND, B.; LÓPEZ-HUERTAS, M. J. Knowledge Organisation. In: KAJBERG, L.; LØRRING, L. (ed). European curriculum reflections on education in library and information science. Copenhagem: Royal School of Library and Information Science, 2005.

CAPURRO, R.; HJØRLAND, B. O conceito de informação. Perspectivas em Ciência da Informação, Belo Horizonte, v. 12, n. 1, p. 148 - 207, jan. / abr. 2007.

CHAUÍ, M. Convite à Filosofia. 13. ed. São Paulo: Ática, 2009.

DUBOIS, J.; GIACOMO, M; GUESPIN, L.; MARCELLESI, C.; MARCELLESI, JB.; Mevel, J-B.Dicionário de linguística. São Paulo: Cultrix, 1973. 653 p.

FRIEDMAN, A.; THELLEFSEN, M. Concept theory and semiotics in knowledge organization. Journal of documentation, v. 67, n. 4, 2011. p. $644-674$.

GARDIN, J-C. Document analysis and linguistic theory. Journal of documentation, v. 29, n. 2, jun. 1973.

HODGE, G. Systems of knowledge organization for digital libraries: beyond traditional authority files, 2000. Disponível em: www.clir.org/pubs/reports/ pub91/contents.html.

HJØRLAND, B. What is Knowledge Organization (KO)? Knowledge Organization, v. 35, n. 2/3, p. $86-101,2008$.

INGWERSEN, p. Conceptions of Information Science. In: VAKKARI, P.; CRONIN, B. (ed.). Conceptions of Library and Information Science: 
historical, empirical and theoretical perspectives. London: Taylor Graham, 1992. p. $299-312$.

KOBASHI, N. Y.; TÁLAMO, M. de F. G. M. Informação: fenômeno e objeto de estudo da sociedade contemporânea. Transinformação, Campinas, SP, v. 15, n. especial, p. $7-21$, set. / dez. 2003.

LARA, M. L. G. de. A construção da informação no universo da linguagem na contemporaneidade. In: LARA, M. L. G. de; FUJINO, A.; NORONHA, D. P. (org.) Informação e contemporaneidade: perspectivas. Recife, PE: Néctar, 2007. p. $153-168$.

LE COADIC, Y. A ciência da informação. Brasília: Briquet de Lemos, 2004.

LOPES, E. Fundamentos da linguística contemporânea. 13. ed. São Paulo: Cultrix, 1993.

LYONS, J. Semântica - I. Lisboa: Editorial Presença, 1977.

MAIMONE, G. D. Postmodernidad en la Ciencia de la Información: contextos interdisciplinares. Saarbrücken, Germany: Editorial Académica Española, 2012. 66 p.

PETTER, M. Linguagem, língua, lingüística. In: FIORIN, J. L. (org.). Introdução à Lingüística: 1. Objetos teóricos. 3. ed. São Paulo: Contexto, 2003. p. 11-24.

ROQUETA, M. B. Sistemas de organización del conocimiento: uma tipologia actualizada. Informação \& Informação, Londrina, v. 16, n. 3, p. 122 - 139, jan./jun. 2011.

SARACEVIC, T. Relevance: a view of and a framework for the thinking on the notion in Information Science. Journal of the American Society for Information Science, Nov./Dec. 1975.

SVENONIUS, E. The intelectual foundation of information organization. Cambridge, Massachusetts; London, England: The MIT Press, 2001.

TÁLAMO, M. de F. G. M. Linguagem documentária. São Paulo: APB, 1997.

TÁLAMO, M. de F. G. M.; LARA, M. L. G. de. O campo da linguística documentária. Transinformação, Campinas, v. 18, n. 3, p. $203-211$, set. / dez. 2006.

ZINS, C. Knowledge organization: an epistemological perspective. Knowledge Organization, v. 31, n. 1, p. $49-54,2004$. 


\title{
INFORMATION AND KNOWLEDGE: INPUTS FOR ORGANIZATIONAL WORK IN INFORMATION SCIENCE
}

\begin{abstract}
Introduction: Conceptualize information and knowledge in the field of Information Science to elucidate the importance they acquire when integrating Knowledge Organization Systems (SOC). Objective: To show the importance of information and knowledge concepts for SOCs. Methodology: Survey and analysis of theoretical framework on the theme of knowledge organization information and knowledge organization systems (SOC). Results: Information and knowledge are fundamental elements for the elaboration of knowledge organization systems (SOC) because information is a communicative vehicle in the social sphere and knowledge, in addiction to begin a carrier of scientific and technological development, when registered is liable to be transformed into information, documentary. SOCs are structured in controlled languages, tools purposely designed to acess information. Conclusions: All organization systems in Information Science necessarily passes through some type of language that can provide information retrieval to become knowledge.
\end{abstract}

Descriptors: Information. Knowledge. Knowledge Organization Systems.

\section{INFORMACIÓN Y CONOCIMIENTO: ENTRADAS PARA EL TRABAJO ORGANIZACIONAL EN CIENCIA DE LA INFORMACIÓN}

\begin{abstract}
RESUMEN
Introducción: Conceptualiza la información y el conocimiento em el campo de la Ciencia de la Información para dilucidar la importância que adquieren al integrar los Sistemas de Organización del Conocimiento (SOC). Objetivo: Destacar la importância de los conceptos de información y conocimiento para los SOC. Metodología:. Lavantamiento y análisis del marco teórico sobre el tema de la organización del conocimiento y da información y los sistemas de organización del conocimiento (SOC). Resultados: La información y el conocimiento son elementos fundamentales para la elaboración de sistemas de organización del conocimiento (SOC) porque la información es um vehículo comunicativo em la esfera social y el conocimient, además de ser um portador del desarrollo científico y tecnológico, cuando está registrado puede transformarse em información, documental. Los SOC están estructurados em lenguages controlados, herramientas diseñadas especificamente para aceder a la información. Conclusiones: Todo el sistema organizacional em Ciencia de la Información passa necessariamente por algún tipo de lenguage que puede proporcionar la recuperación de información para convertirse en conocimiento.
\end{abstract}

Descriptores: Información. Conocimiento. Sistemas de Organización del Conocimiento.

Recebido em: 15/09/2020

Aceito em: 30/09/2020 